\title{
The Suicidal Ideation Attributes Scale (SIDAS): Community-Based Validation Study of a New Scale for the Measurement of Suicidal Ideation
}

\author{
Bregje A. J. van Spijker, PhD, Philip J. Batterham, PhD, Alison L. Calear, PhD, \\ Louise Farrer, PhD, Helen Christensen, PhD, Julia Reynolds, MPsych(Clin), and \\ Ad J. F. M. Kerkhof, PhD
}

While suicide prevention efforts are increasingly being delivered using technology, no scales have been developed specifically for web-based use. The Suicidal Ideation Attributes Scale (SIDAS) was developed and validated as a brief, web-based measure for severity of suicidal ideation, using an online survey of Australian adults $(n=1,352)$. The SIDAS demonstrated high internal consistency and good convergent validity. Frequency and controllability of thoughts were more strongly associated with suicide plans and attempts than other attributes assessed. Scores $\geq 21$ indicated high risk of suicide behavior. The SIDAS appears to be a valid web-based measure for severity of suicidal ideation.

Bregje A. J. van Spijker, Centre for Mental Health Research, The Australian National University, Canberra, Australia, and Black Dog Institute, University of New South Wales, Sydney, Australia; Philip J. Batterham, Alison L. Calear, and Louise Farrer, Centre for Mental Health Research, The Australian National University, Canberra, Australia; Helen ChrisTENSEN, Black Dog Institute, University of New South Wales, Sydney, Australia; Julia ReYnolds, Centre for Mental Health Research, The Australian National University, Canberra, Australia; AD J.F.M. KerkHOF, Department of Clinical Psychology and the EMGO+ Institute for Health and Care Research, Faculty of Psychology and Education, VU University Amsterdam, Amsterdam, The Netherlands.

The study was funded by a fellowship support award from the College of Medicine, Biology and Environment, The Australian National University. This research also forms part of research conducted by the NHMRC Centre for Research Excellence in Suicide Prevention. PB is supported by NHMRC Early Career Fellowship 1035262. ALC is supported by NHMRC Early Career Fellowship 1013199. HC is supported by NHMRC Senior Principal Research Fellowship 525411.

Address correspondence to B. van Spijker, Centre for Mental Health Research, The Australian National University, Building 63, Eggleston Road, Acton, ACT 0200, Australia; E-mail: baj. van.spijker@gmail.com
Suicidal ideation is a significant problem that causes significant burden (van Spijker, van Straten, Kerkhof, Hoeymans, \& Smit, 2011). However, ideation is usually measured as a dichotomous outcome in epidemiological trials, which says little about the severity of the ideation or about other attributes, such as controllability. Moreover, while both research and suicide prevention activities are increasingly being delivered over the Internet and through mobile phone applications, there are no suicidal ideation instruments developed specifically for these media or validated primarily for use in the online setting. A number of scales such as the Scale for Suicide Ideation (SSI; Beck \& Steer, 1991) and the Columbia Suicide Severity Rating Scale (C-SSRS; Posner et al., 2011) provide more extensive information, but these are often not suited to an epidemiological or online context because they are costly in research, were developed and validated as a clinical interview, require administration by a clinically trained individual, or are not sufficiently concise in the context of administration within a comprehensive epidemiological 
assessment. Similarly, the majority of brief screeners that are available would not be sensitive to change as they rely on binary responses (Allison, Hubbard, Ginzburg, \& Rachal, 1986; Carroll, Feinberg, Smouse, Rawson, \& Greden, 1981; Centers for Disease Control, 2009; LanghinrichsenRohling, Snarr, Slep, Heyman, \& Foran, 2011; Lindelow, Hardy, \& Rodgers, 1997; Paykel, Myers, Lindenthal, \& Tanner, 1974; World Health Organisation, 1994). Consequently, there has been an absence of investigation in epidemiological studies of the features or attributes of suicidal thoughts such as repetitiveness, invasiveness, and debilitation. As suicidality (defined here as the suicidal continuum ranging from suicidal wishes, thoughts, and plans to behavior and attempts) can occur in a range of psychiatric disorders (Oquendo, BacaGarcia, Mann, \& Giner, 2008), investigating attributes of the thought pattern of those at risk can provide valuable information about transdiagnostic risk profiles. In particular, such investigation may help in identifying specific characteristics of thoughts that play a role in overarching psychiatric disorders associated with suicidality, which may lead to better targeted prevention efforts.

Dissatisfaction with existing suicidal ideation measures for epidemiological purposes and a lack of instruments validated for web-based use were among the reasons for developing an online, brief, freely available, self-report scale that does not require training. For optimal online use, this scale should be able to identify both the lower and the more severe end of the suicidal ideation spectrum. Collected in epidemiological trials, this information could serve to provide tailored advice to consumers looking for appropriate mental health services, including web interventions and online chat services. The current study reports on the development of a new, brief, web-based instrument for the severity of suicidal ideation, the Suicidal Ideation Attributes Scale (SIDAS), which addresses the shortcomings of previous scales. The study validates the SIDAS against the selfreport version of the ideation items from the C-SSRS (Posner et al., 2011) and examines its relationship with indicators of severe suicidality (defined here as plans, preparations, and attempts).

Five attributes of suicidal thoughts were defined and measured. The first two, frequency and controllability of thoughts, were based on the observation that suicidal thoughts are often repetitive and difficult to manage (Kerkhof \& van Spijker, 2011; Morrison \& O'Connor, 2008). Both of these attributes are key elements in the definitions of worry and rumination (Borkovec, DePree, Pruzinsky, \& Robinson, 1983; p. 10; Nolen-Hoeksema, 1991, p. 569) and are acknowledged as potentially relevant dimensions in the overarching process labeled "repetitive thinking" (Watkins, 2008). The third attribute, closeness to attempt, reflects the presence of severe ideation, a condition associated with the emergence of suicidal behaviors (Lewinsohn, Rohde, \& Seeley, 1996; Posner et al., 2011). The last two attributes, level of distress associated with the thoughts and the impact on daily functioning, were included to align the assessment of ideation with other measures of mental disorders that use these as an indication of severity and extent of deviation from normalcy (American Psychiatric Association, 2000; Lecrubier et al., 1997). The item about daily functioning is also useful as a single-item global rating of quality of life (de Boer et al., 2004; Bowling, 2005).

In this article, severe suicidality is classified as plans, preparations, and attempts. We hypothesized that the association with severe suicidality and each of the five attributes would vary. The frequency and controllability of ideation was expected to be less strongly associated with more severe suicidality than attributes more closely linked to behavior; that is, impact on functioning, level of distress, and closeness to attempt. Previous findings show that disability is higher in suicide attempt relative to suicide ideation alone (van Spijker et al., 
2011). We therefore expected impact on functioning to be more strongly associated with severe suicidality. In addition, the suggestion that people attempt suicide to escape intolerable distress (Baumeister, 1990; Nock, Hwang, Sampson, \& Kessler, 2010) led us to hypothesize that levels of distress would be more closely linked to behavior than the frequency and controllability attributes. Finally, because all attributes assess severity of suicidality, strong correlations among the attributes were expected.

\section{METHOD}

\section{Participants and Procedure}

An online survey was developed to examine a range of mental health outcomes, with a particular focus on suicidal ideation. The survey took approximately 30 minutes to complete and included measures assessing psychological distress, depression, anxiety disorders, alcohol use, sleep problems, suicidal ideation, suicide literacy, suicide stigma, exposure to suicide, interpersonal risk factors for suicide, and a range of demographics (Batterham, Calear, \& Christensen, 2013). To reach a diverse segment of the community, participants were recruited through online advertising on the social network site Facebook. Recruitment advertisements were targeted to individuals aged 18 years and older living in Australia. From 12,773 clicks on the advertisements, 1,283 participants fully completed a survey and 610 partially completed a survey, resulting in 1,352 participants who completed the SIDAS and 1,309 who completed all measures included in the present analyses.

All participants were provided with written information about the study aims prior to commencing the survey, and informed consent was given online. Participation in the survey was anonymous. A brief list of state-based mental health service contacts was provided to individuals at the consent stage to assist those participants who might be seeking further help. Participants who completed the survey were provided with an additional list of mental health resources, including physician directory information, help lines, crisis care services, informational resources, and online services. The study received ethics approval from the Science and Medical Delegated Ethics Review Committee at the Australian National University (protocol number 2012/310).

\section{Measures}

Suicidal Ideation Attributes Scale (SI$D A S)$. The SI-DAS is a five-item scale assessing frequency (item 1), controllability (item 2), closeness to attempt (item 3), distress (item 4), and interference with daily activities (item 5) on 10-point scales over the past month. The items are:

1. "In the past month, how often have you had thoughts about suicide?" (0 Never, 1-9: unlabeled points, 10 Always);

2. "In the past month, how much control have you had over these thoughts?" (0 No control/do not control, 1-9: unlabeled points, 10 Full control);

3. "In the past month, how close have you come to making a suicide attempt?" (0 Not at all close, 1-9: unlabeled points, 10 Have made an attempt);

4. "In the past month, to what extent have you felt tormented by thoughts about suicide?" (0 Not at all, 1-9: unlabeled points, 10 Extremely); and,

5. "In the past month, how much have thoughts about suicide interfered with your ability to carry out daily activities, such as work, household tasks or social activities?" (0 Not at all, 1-9: unlabeled points, 10 Extremely).

A 10-point scale was chosen to capture a larger degree of variability than might be expected from fewer categories, 
and to result in an approximately continuous scale. Respondents who endorse a frequency of zero (Never) on the first item of the scale skip the remaining items and are given scores of 10 for controllability (full control) and zero for closeness to attempt, distress, and interference. Total SIDAS scores are calculated as the sum of the five items, with controllability reverse scored $(10=0,9=1, \ldots, 0=10)$, with total scale scores ranging from 0 to 50 . Higher scores are indicative of greater suicidal ideation severity. The SIDAS generally takes between 30 and 60 seconds to complete, although it is substantially shorter for individuals with no suicidal ideation. The scale had strong internal reliability in the current study (Cronbach $\alpha=0.91$ ).

Columbia Suicide Severity Scale (CSSRS). Three ideation items from the C-SSRS - Baseline version (Posner et al., 2011) were measured: frequency, duration, and controllability of thoughts assessed on 5-6-point Likert scales. Two questions from the C-SSRS on the presence of suicidal ideation, three questions about suicide planning, and one question about suicide attempt or preparation were included in the survey. The time frame of the C-SSRS items is past year. The plan and attempt items were used as outcome variables in the present analyses. The C-SSRS ideation subscale has good internal consistency, with Cronbach's $\alpha$ ranging from 0.73 to 0.94 (Posner et al., 2011). Cronbach's $\alpha$ in the current sample was 0.87 . The C-SSRS also has demonstrated convergent, divergent, and predictive validity (Posner et al., 2011). The C-SSRS as used in this study takes between 75 and 120 seconds to complete.

Demographics. Age group, gender, educational status, employment status, marital status, location (metropolitan, regional, rural, or remote), and language spoken at home were assessed using categorical items in the survey.

Patient Health Questionnaire 9 (PHQ-9). The PHQ-9 consists of nine items and was used to assess depression symptoms in the past 2 weeks. Each item is scored $0-3$, and total scores range from 0 to 27 , with higher scores indicating more severe depression symptoms. The internal reliability of the PHQ-9 is excellent with Cronbach's $\alpha$ ranging from 0.86 to 0.89 (Kroenke, Spitzer, \& Williams, 2001). In the current sample, Cronbach's $\alpha$ was 0.92. The PHQ-9 also has established criterion validity, construct validity, and external validity (Kroenke et al., 2001).

Generalized Anxiety Disorder 7 (GAD-7). The GAD-7 was used to assess anxiety in the past 2 weeks. It consists of seven items, each of which is rated $0-3$. Total scores range from 0 to 21 , with higher scores indicating more severe anxiety symptoms. The GAD-7 has good internal consistency (Cronbach's $\alpha=0.92$ ) and testretest reliability, as well as criterion, construct, factorial, and procedural validity (Spitzer, Kroenke, Williams, \& Lowe, 2006). In the current sample, the GAD-7 had a Cronbach's $\alpha$ of 0.93 .

Insomnia Severity Index (ISI). The ISI measured insomnia severity in the past 2 weeks using seven items, which are rated on a 5-point Likert scale. Total scores range from 0 to 28 , with higher scores indicating greater insomnia severity. The ISI has good internal consistency (Cronbach's $\alpha$ ranging from 0.74 to 0.91 ), is sensitive to change, and has demonstrated good convergent, concurrent, and content validity (Bastien, Vallières, \& Morin, 2001; Morin, Belleville, Bélanger, \& Ivers, 2011). The scale also had good internal consistency in the current sample (Cronbach's $\alpha=0.86$ ).

\section{Analysis}

Sample characteristics were reported, and SIDAS total scores were compared between subgroups of the sample using one-way ANOVA. Correlations between SIDAS and C-SSRS items were used to assess the convergent validity of the SIDAS. Similarly, correlations between SIDAS total score and the PHQ-9, GAD-7, and ISI were produced to assess convergent validity. 
Factor analysis was used to examine whether the SIDAS items measured a single construct. The internal consistency of the SIDAS scale was evaluated using Cronbach's $\alpha$. The relationship between each attribute of suicidal ideation with severe suicidality (plans, preparation, and attempts) was assessed using logistic regression. Finally, ROC curves were constructed to assess scale sensitivity and specificity to evaluate whether the SIDAS could distinguish individuals with high risk for suicidal behaviors from those at lower risk using cut points.

\section{RESULTS}

The characteristics of the sample, along with mean SIDAS total scores for the full sample and for each subgroup, are presented in Table 1. Differences in SIDAS total scores were compared using one-way ANOVA, with comparison groups specified in parentheses where necessary. There was high prevalence of recent suicidal ideation (41.4\%) and preparation/attempts (10.7\%) in the sample. Depression, anxiety, and insomnia were all significantly associated with SIDAS total score. Young adults and older adults were well represented in the sample, and females constituted more than $55 \%$ of the sample $(3.1 \%$ were classified as other gender or refused). Two-thirds of the sample was in employment or education, while more than $80 \%$ had at least completed high school. The elevated proportion of single participants $(39.9 \%)$ is likely related to the large group of young adults included in the sample. The majority of participants were living in major cities, although respondents from rural and remote areas $(15.8 \%)$ were slightly overrepresented relative to the Australian population (13.0\%; Australian Bureau of Statistics, 2004). There was also a moderate proportion of the sample (14.9\%) who spoke a language other than English at home.

The distribution of the SIDAS total scale score was positively skewed, with
$50.1 \%$ of participants reporting ideation scores of between 1 and 10 and only $10.4 \%$ scoring between 31 and 50 . In the complete sample, mean SIDAS item scores were 1.42 $(S D=2.39)$ for frequency, 8.58 (2.67) for controllability (reverse scored controllability mean $=1.42), 0.57(1.72)$ for closeness to attempt, 1.19 (2.36) for distress, and 0.90 (2.14) for interference with daily activities. Mean SIDAS total scale scores increased through the spectrum of suicidal thoughts and behaviors from 5.5 in the full sample, to 13.3 in those with ideation, to 43 in those with a recent attempt. Younger people had significantly higher SIDAS scores than older people, $F(2,1310)=23.4$, $p<.001$; however, there was no effect of gender, $F(1,1308)=0.5, p=.827$. Participants who were unemployed or not in the labor force had significantly higher scores, as did students, although this relationship may be confounded with age, $F(3$, $1300)=16.8, p<.001$. High school graduates tended to have lower scores than those with lower or higher education, $F(2$, $1307)=10.7, p<.001$. The high scores of single respondents may be confounded with age; however, the elevated scores of separated or divorced respondents are likely to be independent of age, $F(3,1291)=14.9$, $p<.001$. There was no significant effect of geographical location, $F(2,1310)=0.2$, $p=.810$. Participants who exclusively spoke English at home had significantly higher scores on the SIDAS than those who did not, $F(1,1319)=10.7, p=.001$. Relationships of individual SIDAS items with the subgroups of the sample closely mirrored the relationships seen for the SIDAS total score.

The correlation matrix for items in the SIDAS and the C-SSRS is shown in Table 2. Only participants with any level of suicidal ideation (based on the SIDAS frequency item) were included in this table. The table shows high correlations between items within the SIDAS (shaded area) and between the SIDAS and the C-SSRS ideation items. All absolute correlations were at least 0.3 . The strong correlation (0.66) 
TABLE 1

Sample Characteristics and SIDAS Total Scores for Subgroups of the Sample $(n=1,352)$

\begin{tabular}{|c|c|c|c|c|}
\hline & \multirow[b]{2}{*}{$n(\%)$} & \multicolumn{2}{|c|}{$\begin{array}{l}\text { SIDAS total } \\
\text { score }\end{array}$} & \\
\hline & & Mean & $(S D)$ & \\
\hline Full sample & $1,352(100.0)$ & 5.51 & $(9.79)$ & \\
\hline Suicidal ideation, past month (vs. no ideation) & $560(41.4)$ & 13.30 & $(11.31)$ & ** \\
\hline Suicide plan, past year (vs. no plan) & $220(16.3)$ & 17.25 & $(13.60)$ & ** \\
\hline Suicide preparation/attempt, past year (vs. no attempt) & $144(10.7)$ & 20.70 & $(14.47)$ & ** \\
\hline Suicide attempt, past month (vs. no attempt) & $13(1.0)$ & 42.92 & $(6.16)$ & ** \\
\hline Depression (PHQ-9 criteria vs. no depression) & $322(24.6)$ & 14.47 & $(13.21)$ & ** \\
\hline Generalized anxiety (GAD-7 criteria vs. no anxiety) & $258(19.7)$ & 14.26 & $(13.21)$ & ** \\
\hline Insomnia (ISI criteria vs. no insomnia) & $622(47.8)$ & 8.82 & $(11.69)$ & ** \\
\hline \multicolumn{5}{|l|}{ Age group } \\
\hline $18-24$ & $437(32.3)$ & 7.51 & $(11.56)$ & ** \\
\hline $25-49$ & $390(28.8)$ & 5.73 & $(9.51)$ & \\
\hline $50+$ & $486(35.9)$ & 3.26 & $(7.13)$ & \\
\hline \multicolumn{5}{|l|}{ Gender } \\
\hline Male & $549(40.6)$ & 5.35 & $(9.18)$ & \\
\hline Female & $761(56.3)$ & 5.47 & $(10.06)$ & \\
\hline \multicolumn{5}{|l|}{ Employment } \\
\hline Full-/part-time employed & $572(42.3)$ & 4.44 & $(8.36)$ & ** \\
\hline Unemployed/not in labor force & $241(17.8)$ & 8.44 & $(11.74)$ & \\
\hline Student & $318(23.5)$ & 6.56 & $(11.07)$ & \\
\hline Retired & $173(12.8)$ & 2.57 & $(6.13)$ & \\
\hline \multicolumn{5}{|l|}{ Education } \\
\hline Less than high school & $181(13.4)$ & 6.95 & $(11.13)$ & ** \\
\hline High school graduate & $715(52.9)$ & 4.33 & $(8.41)$ & \\
\hline Post-high school & $414(30.6)$ & 6.74 & $(10.88)$ & \\
\hline \multicolumn{5}{|l|}{ Marital status } \\
\hline Married/de facto & $486(35.9)$ & 3.75 & $(8.05)$ & ** \\
\hline Single, never married & $540(39.9)$ & 7.43 & $(11.19)$ & \\
\hline Separated/divorced & $216(16.0)$ & 5.17 & $(9.07)$ & \\
\hline Widowed & $53(3.9)$ & 2.23 & $(5.92)$ & \\
\hline \multicolumn{5}{|l|}{ Location } \\
\hline Metropolitan city & $740(54.7)$ & 5.54 & $(9.88)$ & \\
\hline Regional area & $359(26.6)$ & 5.16 & $(9.14)$ & \\
\hline Rural or remote area & $214(15.8)$ & 5.58 & $(10.03)$ & \\
\hline \multicolumn{5}{|l|}{ Language } \\
\hline Speak only English at home & $1,119(82.8)$ & 5.84 & $(9.97)$ & * \\
\hline Speak non-English language at home & $202(14.9)$ & 3.41 & $(8.29)$ & \\
\hline
\end{tabular}

SIDAS, Suicidal Ideation Attributes Scale; not all variables add to $100 \%$ due to "refused" responses. ${ }^{* *} p<.001 ; *_{p}<.05$.

between the frequency item of the SIDAS and the frequency item of the C-SSRS is indicative of convergent validity. The correlation between the controllability items from the two scales was smaller; however, this may be related to the wording of the questions ("how much control have you had..." in the SIDAS vs. "could you stop thinking about...") or the different time frames (past month vs. past year). Nevertheless, the strong correlations between the SIDAS total scale score and C-SSRS items 
suggest that the scales measure the same underlying construct. Similarly, the SIDAS showed generally strong correlations with the PHQ-9 total score (0.65), GAD-7 total score $(0.58)$, and ISI (0.40).

Two-factor analyses were conducted: one including all respondents and one only including participants who reported ideation. The factor loadings for both analyses are in Table 3. In the full sample, the single factor had an eigenvalue of 3.8 and accounted for $75.5 \%$ of the total variance, while in the ideation sample, the single factor had an eigenvalue of 3.3 and accounted for $65.5 \%$ of the total variance. Cronbach's $\alpha$ was 0.91 for the full sample and 0.86 among those with ideation, indicating high internal consistency in both cases. All items had absolute factor loadings greater than 0.6 (controllability has reverse coding). Consequently, the diverse attributes of ideation measured in the SIDAS appear to contribute to a unidimensional construct of suicidal ideation.

The relationship between each attribute of ideation and plans and attempts was examined using logistic regression analyses (Table 4). The outcome variables of plans and attempts were items taken from the CSSRS. As closeness to acting on ideation (SIDAS item 3) may be confounded with attempts (as suggested in Table 4 by the significant, large odds ratio for this item), models were run with and without this attribute. Nevertheless, the two models had very similar findings. Greater frequency of thoughts and less controllability of thoughts were strongly associated with increased odds of preparation/attempt or suicide plan in the past year. Specifically, every one point increase in SIDAS frequency score was associated with a $26 \%$ increase in the odds of preparation/attempt and 25\% increase in the odds of suicide plan in the past year, based on the second model. One point decreases in SIDAS controllability were associated with $18 \%$ increases in both preparation/attempt. Levels of SIDAS distress and interference with daily activities were not significantly associated with plans 
TABLE 3

Factor Analysis of the Suicidal Ideation Attributes Scale for the Complete Sample and among Participants with Ideation: Factor Loadings

\begin{tabular}{lcc}
\hline & Complete sample $(n=1,352)$ & Participants with ideation $(n=560)$ \\
\hline Frequency & 0.90 & 0.83 \\
Controllability & -0.79 & -0.63 \\
Closeness to acting & 0.83 & 0.82 \\
Distress & 0.91 & 0.87 \\
Interfere with daily activities & 0.90 & 0.88 \\
Eigenvalue & 3.8 & 3.3 \\
Variance accounted for & $75.5 \%$ & $65.5 \%$ \\
Cronbach's Alpha & 0.91 & 0.86 \\
\hline
\end{tabular}

TABLE 4

Logistic Regression Models of the Relationship between Attributes of Ideation and Suicide Attempts or Plans in the Past Year $(n=1,287)$

\begin{tabular}{|c|c|c|c|c|c|c|c|c|}
\hline & \multicolumn{4}{|c|}{ Preparation/attempt in past year } & \multicolumn{4}{|c|}{ Suicide plan in past year } \\
\hline & \multicolumn{2}{|c|}{ All attributes } & \multicolumn{2}{|c|}{$\begin{array}{l}\text { Excluding } \\
\text { closeness to } \\
\text { acting }\end{array}$} & \multicolumn{2}{|c|}{ All attributes } & \multicolumn{2}{|c|}{$\begin{array}{l}\text { Excluding } \\
\text { closeness to } \\
\text { acting }\end{array}$} \\
\hline & OR & $p$ & OR & $p$ & OR & $p$ & OR & $p$ \\
\hline Frequency & 1.151 & .024 & 1.261 & $<.001$ & 1.176 & .003 & 1.245 & $<.001$ \\
\hline Controllability & 0.869 & .001 & 0.850 & $<.001$ & 0.866 & $<.001$ & 0.849 & $<.001$ \\
\hline Closeness to acting & 1.601 & $<.001$ & & & 1.428 & $<.001$ & & \\
\hline Distress & 1.099 & .188 & 1.094 & 145 & 1.085 & .196 & 1.099 & .099 \\
\hline Interfere with daily activities & 0.901 & .150 & 1.074 & 205 & 0.983 & .780 & 1.086 & .124 \\
\hline Age group & & .903 & & .719 & & .211 & & .333 \\
\hline $18-24$ & 1.000 & & 1.000 & & 1.000 & & 1.000 & \\
\hline $25-49$ & 0.874 & .661 & 0.807 & .452 & 1.389 & .211 & 1.247 & .381 \\
\hline $50+$ & 0.882 & .741 & 0.785 & .504 & 1.724 & .079 & 1.565 & .139 \\
\hline \multicolumn{9}{|l|}{ Gender } \\
\hline Female & 1.000 & & 1.000 & & 1.000 & & 1.000 & \\
\hline Male & 0.937 & .780 & 0.806 & .331 & 1.512 & .027 & 1.361 & .090 \\
\hline Marital status & & .456 & & .330 & & .009 & & .010 \\
\hline Married/de facto & 1.000 & & 1.000 & & 1.000 & & 1.000 & \\
\hline Single, never married & 1.597 & .153 & 1.600 & .127 & 1.869 & .020 & 1.831 & .021 \\
\hline Separated/divorced & 1.357 & .402 & 1.454 & .278 & 2.281 & .002 & 2.247 & .002 \\
\hline Widowed & 0.611 & .639 & 0.508 & .523 & 1.592 & .374 & 1.458 & .478 \\
\hline
\end{tabular}

Bold values indicate $p<.05$.

or attempts. The models controlled for age, gender, and marital status, with only one significant association being found between marital status and plans of suicide. Single participants had an $83 \%$ increase in the odds of reporting a suicide plan in the past year, while the odds were increased by a factor of 2.3 in those who were separated or 
divorced. These effects were smaller and not significant for preparation/attempt.

To evaluate whether the SIDAS could distinguish individuals at high risk of suicidal behaviors from those at lower risk, ROC curves were constructed to assess scale sensitivity and specificity. A cut point of 1 had sensitivity of $85.5 \%$ for suicide plans and $84.0 \%$ for suicide attempts (with $67.1 \%$ and $63.6 \%$ specificity, respectively). However, high specificity is required to identify individuals most likely to engage in suicidal behavior. Scores $\geq 21$ had $95.8 \%$ specificity for presence of plan in the past year and $94.9 \%$ specificity for presence of preparation/attempt in the past year (with $39.6 \%$ and $50.0 \%$ sensitivity, respectively). This level of ideation severity was seen in approximately $25 \%$ of the present sample. Based on these results, any ideation would be indicative of risk for suicidal behavior while a cutoff of 21 on the SIDAS may be used to indicate high risk of suicide behavior. The rates of plans and attempts among those with no ideation (SIDAS score 0), low ideation (score 1-20), and high ideation (score 21-50) are shown in Table 5. Rates of plans increase sixfold between no and low ideation severity and nearly threefold between low and high severity groups. Likewise, attempt rates increase fourfold and nearly fivefold, respectively, across nolow and low-high severity groups.

\section{DISCUSSION}

The SIDAS demonstrated strong internal consistency and good convergent validity, with one factor accounting for the majority of the variance. Contrary to our hypothesis, the SIDAS frequency and controllability items were more strongly associated with a history of severe suicidality than the other attributes, indicating their potential greater predictive utility. This could be further established using a longitudinal design. Recent developments exploring the similarities between suicidal ideation and repetitive thinking, in which frequency and controllability play a significant role, are consistent with this finding (Kerkhof \& van Spijker, 2011). Any ideation was found to increase the risk of suicidal behavior, and a score $\geq 21$ indicated high risk of suicide behavior. If further validation proved consistent, this cutoff might be used to detect severe ideation and high suicide risk in the general population.

The internal consistency of the SIDAS was similar to widely used scales such as the C-SSRS (Posner et al., 2011) and the SSI (Beck, Brown, \& Steer, 1997; Beck, Kovacs, \& Weissman, 1979). The significant associations with depression, anxiety, and insomnia provide additional support for the validity of the SIDAS. Test-retest reliability and sensitivity to change could not be determined due to the cross-sectional nature of the current study. Future research would need to establish these indicators. However, it is likely that the SIDAS will be more sensitive to change than binary scales because it measures different levels of severity.

Although the SIDAS has the advantage of being a brief, time-efficient, webbased measure, several limitations regarding the scale and this study should be noted. First, there may be other attributes not

TABLE 5

Relationship between SIDAS Scores and Suicidal Behaviors in the Past Year $(n=1,352)$

\begin{tabular}{lccc}
\hline SIDAS score & $n$ & $\begin{array}{c}\text { Suicide plan in past } \\
\text { year }\end{array}$ & $\begin{array}{c}\text { Preparation/attempt in past } \\
\text { year }\end{array}$ \\
\hline 0 (no ideation) & $792(41.8 \%$ of sample) & $32(4.0 \%)$ & $23(2.9 \%)$ \\
$1-20$ (low ideation) & $426(76.1 \%$ of ideators) & $101(23.7 \%)$ & $49(11.5 \%)$ \\
$21-50$ (high & $134(23.9 \%$ of ideators) & $87(64.9 \%)$ & $72(53.7 \%)$ \\
ideation) & & & \\
\hline
\end{tabular}


included in the current scale that are associated with the severity of ideation and with more severe suicidality (plans, preparations, and attempts), such as the duration of thoughts (Posner et al., 2011). Similarly, there may be other features of ideation that predict risk or additional risk factors that distinguish subtypes of ideation, such as desire to live, hopelessness, perfectionism, and burdensomeness (Beck, Brown, Berchick, Stewart, \& Steer, 1990; Brown, Steer, Henriques, \& Beck, 2005; O'Connor, 2007; van Orden et al., 2010). These have all been shown to be of relevance to suicidality, but were not included in the SIDAS in the interests of brevity. The wording of the first item ("In the past month, how often have you had thoughts about suicide/") might result in false positives for a small percentage of people bereaved by suicide or people working in suicide prevention who have relatively innocuous thoughts about suicide in a literal sense, but not necessarily about harming themselves. The data do not support this potential misinterpretation, however, as evident from the strong correlations between this item and the subsequent items, and the strong single-factor solution. Still, this potential issue should be taken into account in future research into the SIDAS.

The "closeness to acting on thoughts" attribute may be strongly confounded with behaviors. However, the conclusion that frequency and controllability were strongly associated with increased odds of past suicidal behavior is not compromised by this association. Furthermore, no definition of suicide attempt was provided to respondents, so they would have rated this item according to their own definition of what constitutes a suicide attempt and their personal views on how close they came to making a suicide attempt. Future research could explore how this relates to definitions used in research and clinical settings. Recruitment using Facebook may have affected the representativeness of the sample due to self-selection, but for the purpose of validating the scale, it was important to have high rates of suicidal ide- ation in the sample, while maintaining a focus on the general population rather than a clinical population. As outlined in a separate manuscript (Batterham, 2014), the online sample used in this study showed higher psychopathology than would be expected in the general population, but was still similar in representativeness to comparable postal surveys relative to the Australian population. In addition, the SIDAS was embedded in a large survey, and it is unknown to what extent the rest of the survey might have influenced responses. Finally, the cross-sectional design did not allow prospective examination of predictive associations between ideation and behaviors.

Strengths of the current study include the community-based sample with high numbers of respondents experiencing recent suicidal ideation, plans, and attempts. The SIDAS can therefore be used to collect more detailed information about suicidal thoughts in epidemiological studies. In addition, the fact that the SIDAS is a web-based instrument fits in with recent developments in suicide prevention to increasingly use the Internet in delivering prevention efforts (Pietrzak \& McLaughlin, 2009; van Spijker, Majo, Smit, van Straten, \& Kerkhof, 2012). The SIDAS could therefore be valuable in web-based prevention and intervention research as no other instruments have as yet been developed and validated specifically for online use to our knowledge. Finally, the SIDAS could be useful as an online self-test where different total scores would result in different treatment advice. For example, with appropriate safeguards, someone with low scores might be offered a web-based program to reduce their suicidal thoughts, while another with a high score indicating high risk could be immediately linked with crisis services (face to face, telephone, or web-based). More research is needed to further develop and validate the SIDAS for these uses. In particular, the cutoff score indicating high risk needs further investigation, as well as validation in different populations. More 
generally, the SIDAS may benefit from further validation using independent clinical ratings of the suicidality of respondents.
Overall, the SIDAS appears to be a valid measure for assessing severity of suicidal ideation using the Internet.

\section{REFERENCES}

Allison, M., Hubbard, R. L., Ginzburg, H. M., \& RaChaL, J. (1986). Validation of a three-item measure of depressive and suicidal symptoms. Hospital \& Community Psycbiatry, 37, 738-740.

American Psychiatric Association. (2000). Diagnostic and statistical manual of mental disorders (4th ed., text rev.). Washington, DC: Author.

Australian Bureau of Statistics. (2004). 1301.0 - Year book Australia. Canberra, Australia: Author.

Bastien, C. H., Vallières, A., \& Morin, C. M. (2001). Validation of the Insomnia Severity Index as an outcome measure for insomnia research. Sleep Medicine, 2, 297-307.

Batterham, P. J. (2014). Recruitment of mental health survey participants using internet advertising: Content, characteristics and costeffectiveness. International Fournal of Methods in Psychiatric Research, 23, 184-191.

Batterham, P., Calear, A., \& ChristenSEN, H. (2013). Correlates of suicide stigma and suicide literacy in the community. Suicide and Life-Threatening Behavior, 43, 406-417.

Baumeister, R. F. (1990). Suicide as escape from self. Psychological Review, 97, 90-113.

Beck, A. T., Brown, G., Berchick, R. J., Stewart, B., \& Steer, R. A. (1990). Relationship between hopelessness and ultimate suicide: A replication with psychiatric outpatients. American fournal of Psychiatry, 147, 190-195.

Beck, A. T., Brown, G. K., \& SteEr, R. A. (1997). Psychometric characteristics of the Scale for Suicide Ideation with psychiatric outpatients. Behaviour Research and Therapy, 35, 1039-1046.

Beck, A. T., Kovacs, M., \& Weissman, A. (1979). Assessment of suicidal intention: The Scale for Suicide Ideation. Fournal of Consulting and Clinical Psychology, 47, 343-352.

Beck, A. T., \& Steer, R. A. (1991). Manual for the Beck Scale for Suicide Ideation. San Antonio: The Psychological Corporation.

DE Boer, A. G. E. M., van Lanschot, J. J. B., Stalmeier, P. F. M., van Sandick, J. W., Hulscher, J. B. F., de Haes, J. C. J. M., et al. (2004). Is a single-item visual analogue scale as valid, reliable and responsive as multi-item scales in measuring quality of life? Qualify of Life Research, 13, 311-320.

Borkovec, T. D., DePree, J. A., PruzinSKY, T., \& RobINSON, E. (1983). Preliminary exploration of worry: Some characteristics and processes. Behaviour Research and Therapy, 21, 916.

Bowling, A. (2005). Just one question: If one question works, why ask several? Fournal of Epidemiology and Community Health, 59, 342-345.

Brown, G. K., Steer, R. A., Henriques, G. R., \& BECK, A. T. (2005). The internal struggle between the wish to die and the wish to live: A risk factor for suicide. American Fournal of Psychiatry, 162, 1977-1979.

Carroll, B. J., Feinberg, M., Smouse, P. E., Rawson, S. G., \& Greden, J. F. (1981). The Carroll Rating Scale for Depression, I: Development, reliability and validation. British Fournal of Psychiatry, 138, 194-200.

Centers for Disease Control. (2009). Youth risk behavior survey. Retrieved August 15, 2013, from http://www.cdc.gov/HealthyYouth/ yrbs/questionnaire_rationale.htm.

Kerkhof, A. J. F. M., \& van SpijKer, B. A. J. (2011). Worrying and rumination as proximal risk factors for suicidal behaviour. In R. C. O'Connor, S. Platt, \& J. Gordon (Eds.), International handboook of suicide prevention: Research, policy and practice (pp. 199-209). Chichester: Wiley-Blackwell.

Kroenke, K., Spitzer, R. L., \& WilLIAMS, J. B. W. (2001). The PHQ-9: Validity of a brief depression severity measure. Fournal of General Internal Medicine, 16, 606-613.

Langhinrichsen-Rohling, J., Snarr, J. D., Slep, A. M., Heyman, R. E., \& Foran, H. M. (2011). Risk for suicidal ideation in the U.S. Air Force: An ecological perspective. Fournal of Consulting and Clinical Psychology, 79, 600-612.

Lecrubier, Y., Sheehan, D. V., Weiller, E., Amorim, P., Bonora, I., Harnett Sheehan, K., ET AL. (1997). The Mini International Neuropsychiatric Interview (MINI), A short diagnostive structured interview: Reliability and validity according to the CIDI. European Psycbiatry, 12, 224-231.

Lewinsohn, P. M., Rohde, P., \& Seeley, J. R. (1996). Adolescent suicidal ideation and attempts: Prevalence, risk factors, and clinical implications. Clinical Psychology, 3, 25-46.

Lindelow, M., Hardy, R., \& Rodgers, B. (1997). Development of a scale to measure symptoms of anxiety and depression in the gen- 
eral UK population: The Psychiatric Symptom Frequency Scale. Journal of Epidemiology and Community Health, 51, 549-557.

Morin, C. M., Belleville, G., Bélanger, L., \& Ivers, H. (2011). The Insomnia Severity Index: Psychometric indicators to detect insomnia cases and evaluate treatment response. Sleep, 34, 601-608.

Morrison, R., \& O'Connor, R. C. (2008). A systematic review of the relationship between rumination and suicidality. Suicide and Life-Threatening Behavior, 38, 523-538.

Nock, M. K., Hwang, I., Sampson, N. A., \& Kessler, R. C. (2010). Mental disorders, comorbidity and suicidal behavior: Results from the national comorbidity survey replication. Molecular Psychiatry, 15, 868-876.

Nolen-Hoeksema，S. (1991). Responses to depression and their effects on the duration of depressive episodes. Fournal of Abnormal Psychology, 100, 569-582.

O'Connor, R. C. (2007). The relationship between perfectionism and suicidality: A systematic review. Suicide and Life-Threatening Behavior, 37, 698-714.

Oquendo, M. A., Baca-Garcia, E., ManN, J. J., \& Giner, J. (2008). Issues for DSM-V: Suicidal behavior as a separate diagnosis on a separate axis. American fournal of Psychiatry, 165, 1383-1384.

van Orden, K. A., Witte, T. K., Cukrowicz, K. C., Braithwaite, S. R., Selby, E. A., \& JoIner, T. E. (2010). The interpersonal theory of suicide. Psychological Review, 117, 575600 .

Paykel, E. S., Myers, J. K., Lindenthal, J. J., \& Tanner, J. (1974). Suicidal feelings in the general population: A prevalence study. British Fournal of Psychiatry, 124, 460-469.
Pietrzak, E., \& McLaughlin, R. (2009). The effectiveness of online suicide prevention programs: 1998-2009. A literature review. Brisbane: University of Queensland-Centre for Military and Veterans Health.

Posner, K., Brown, G. K., Stanley, B., Brent, D. A., Yershova, K. V., Oquendo, M. A., ET AL. (2011). The Columbia-Suicide Severity Rating Scale: Initial validity and internal consistency findings from three multisite studies with adolescents and adults. American fournal of Psychiatry, 168, 1266-1277.

van SpIJKer, B. A. J., Majo, C. M., SMit, F., van Straten, A., \& Kerkhof, A. J. F. M. (2012). Reducing suicidal ideation: Cost-effectiveness analysis of a randomized controlled trial of unguided web-based self-help. Fournal of Medical Internet Research, 14, e141.

van Spijker, B. A. J., van Straten, A., Kerkhof, A. J. F. M., Hoeymans, N., \& Smit, F. (2011). Disability weights for suicidal thoughts and non-fatal suicide attempts. Fournal of Affective Disorders, 134, 341-347.

Spitzer, R. L., Kroenke, K., Williams, J. B., \& Lowe, B. (2006). A brief measure for assessing generalized anxiety disorder: The GAD7. Archives of Internal Medicine, 166, 1092-1097.

Watkins, E. R. (2008). Constructive and unconstructive repetitive thought. Psychological Bulletin, 134, 163-206.

World Health Organization. (1994). A user's guide to the self-reporting questionnaire (SRQ). Retrieved August 15, 2013, from http:// apps.who.int/iris/bitstream/10665/61113/1/WHO_ MNH_PSF_94.8.pdf.

Manuscript Received: June 14, 2013

Revision Accepted: October 2, 2013 